practices reported increases in BSIs. Ultimately, our understanding of the impact of the COVID-19 pandemic on HAIs will be limited, due in part to the relaxation of mandatory reporting requirements during the pandemic. ${ }^{1}$ Data on healthcare-associated noncentral-catheter-associated BSI rates will be particularly limited because reporting in the United States is not mandated.

Currently, the full scope of the impact of COVID-19 on HAIs is unclear. However, important opportunities exist for health systems (both on the local level and nationally) to utilize their experiences during the current pandemic to bolster infrastructure and to robustly prepare for and quickly respond to future pandemic threats.

\section{Acknowledgments.}

Financial support. No financial support was provided relevant to this article.

Conflicts of interest. All authors report no conflicts of interest relevant to this article.

\section{References}

1. Stevens MP, Doll M, Pryor R, Godbout E, Cooper K, Bearman G. Impact of COVID-19 on traditional healthcare-associated infection prevention efforts. Infect Control Hosp Epidemiol 2020;41:946-947.
2. Wee LE, Conceicao EP, Tan JY, et al. Unintended consequences of infection prevention and control measures during COVID-19 pandemic. Am J Infect Control 2020. doi: 10.1016/j.ajic.2020.10.019.

3. LeRose J, Sandhu A, Polistico J, et al. The impact of COVID-19 response on central-line-associated bloodstream infections and blood culture contamination rates at a tertiary-care center in the greater Detroit area. Infect Control Hosp Epidemiol 2020. doi: 10.1017/ice.2020.1335.

4. Buetti N, Ruckly S, Montmollin E de, et al. COVID-19 increased the risk of ICU-acquired bloodstream infections: a case-cohort study from the multicentric OUTCOMEREA network. Intens Care Med 2021;47:180-187.

5. Bentivegna E, Alessio G, Spuntarelli V, et al. Impact of COVID-19 prevention measures on risk of health care-associated Clostridium difficile infection. Am J Infect Control 2020. doi: 10.1016/j.ajic.2020.09.010.

6. Ponce-Alonso M, Fuente JS de la, Rincón-Carlavilla A, et al. Impact of the coronavirus disease 2019 (COVID-19) pandemic on nosocomial Clostridioides difficile infection. Infect Control Hosp Epidemiol 2020. doi: 10.1017/ice.2020.454.

7. Sturdy A, Basarab M, Cotter M, et al. Severe COVID-19 and healthcareassociated infections on the ICU: time to remember the basics? J Hosp Infect 2020;105:593-595.

8. National survey shows healthcare facilities implementing PPE crisis standards of care. Association for Professionals in Infection Control and Epidemiology website. https://apic.org/news/national-survey-shows-healthcarefacilities-implementing-ppe-crisis-standards-of-care/. Published December 2020. Accessed February 8, 2021.

\title{
Outbreak of endemic carbapenem-resistant Acinetobacter baumannii in a coronavirus disease 2019 (COVID-19)-specific intensive care unit
}

\author{
Danielle Rosani Shinohara MSc${ }^{1}$, Silvia Maria dos Santos Saalfeld MSc ${ }^{1,2}$, Hilton Vizzi Martinez $\mathrm{MSc}^{2}$, \\ Daniela Dambroso Altafini MSc ${ }^{1,2}$, Bruno Buranello Costa $\mathrm{MSc}^{2}$, Nayara Helisandra Fedrigo $\mathrm{MSc}^{1}$ and \\ Maria Cristina Bronharo Tognim $\mathrm{PhD}^{1}$ (1) \\ ${ }^{1}$ Department of Basic Health Sciences, State University of Maringá, Maringá, Paraná, Brazil and ${ }^{2}$ Maringá University Hospital, State University of Maringá, \\ Maringá, Paraná, Brazil
}

To the Editor-Mechanical ventilation is part of the supportive care arsenal used for patients admitted to intensive care units (ICUs). Currently, with the worldwide coronavirus disease 2019 (COVID-19) pandemic, many patients present severe pulmonary symptoms, and the use of mechanical ventilation has increased dramatically. ${ }^{1}$ Although life saving, mechanical ventilation use can lead to ventilator-associated pneumonia (VAP), with high mortality rates, especially when multidrug-resistant bacteria (eg, Acinetobacter baumannii) are involved.,

Cases of A. baumannii infection were recently reported in COVID-19 patients. $^{3,4}$ In Iran, A. baumannii comprised $90 \%$ of coinfections with severe acute respiratory coronavirus virus 2 (SARS-CoV-2), with mortality rates up to $100 \% .^{3}$ In Israel, Gottesman et $\mathrm{al}^{4}$ described an outbreak (5 cases) of carbapenemresistant A. baumannii (CRAb) in 2 wards of a COVID-19 hospital. To the best of our knowledge, ours is the first study to report a

\footnotetext{
Author for correspondence: Maria Cristina Bronharo Tognim, E-mail: mcbtognim@ uem.br

Cite this article: Shinohara DR, et al. (2022). Outbreak of endemic carbapenemresistant Acinetobacter baumannii in a coronavirus disease 2019 (COVID-19)-specific intensive care unit. Infection Control \& Hospital Epidemiology, 43: 815-817, https:// doi.org/10.1017/ice.2021.98
}

monoclonal outbreak of an endemic CRAb strain in a new COVID-19 ICU, presenting a series of 14 cases.

Due to the COVID-19 pandemic, a tertiary teaching hospital in southern Brazil expanded the number of beds from 123 to 173 to treat COVID-19 patients. All new beds were physically isolated from the other hospital wards. Of the new beds, 20 were in an ICU with 2-bed rooms.

The outbreak occurred between September to December 2020 in this new ICU (Fig. 1). Cases of the present study were defined as all patients with positive SARS-CoV-2 RNA by the RT-qPCR method and a positive culture for CRAb. Bacterial identification and antimicrobial susceptibility testing results were obtained with a BD Phoenix automated system (Becton-Dickinson, Franklin Lakes, NJ). All isolates were typed by the enterobacterial repetitive intergenic consensus-PCR (ERIC-PCR) technique. ${ }^{5}$ BioNumerics version 6.5 software (Applied Maths, Sint-Martens-Latem, Belgium) was used to analyze band patterns. Isolates with a Dice similarity coefficient $\geq 0.93$ were classified as belonging to the same cluster.

In total, 14 cases were included in the study (Fig. 1). The mean patient age was 60 years, with male patients predominating (64\%). The median duration of the ICU stay was 24 days (interquartile 


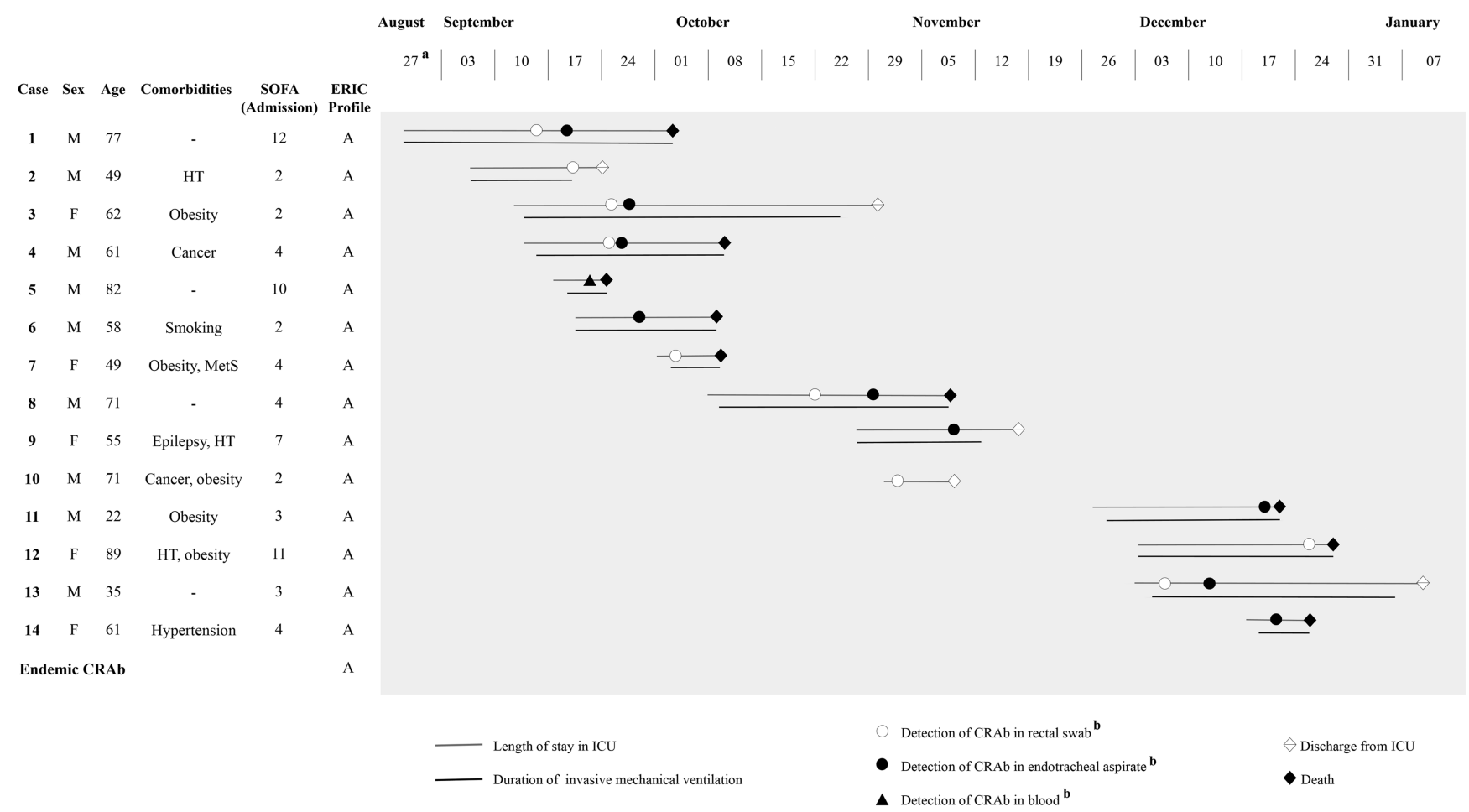

Fig. 1. Schematic description of cases in an outbreak of CRAb in a COVID-19-specific intensive care unit.

${ }^{\text {aT }}$ Temporal representation of cases (weekly).

${ }^{b}$ Date of sample collection for bacterial isolation.

Note. M, male; F, female; SOFA, sequential organ failure assessment score calculated on admission to COVID-19 ICU; ERIC, Enterobacterial Repetitive Intergenic Consensus Polymerase Chain Reaction; HT, hypothyroidism; MetS, metabolic syndrome; CRAb, carbapenem-resistant Acinetobacter baumannii; ICU, COVID-19 intensive care unit.

range [IQR], 14-34), duration of invasive mechanical ventilation was 25 days (IQR, 11-32), and Sequential Organ Failure Assessment score on admission to ICU was 4 (IQR, 3-9).

Overall, 19 CRAb isolates were recovered from blood, endotracheal aspirates, and/or rectal swabs of 14 patients. Of these patients, 13 received invasive mechanical ventilation, 9 were diagnosed with VAP and 1 with bacteremia. Among the 9 patients with a CRAb-positive rectal swabs, 5 also had CRAb-positive endotracheal aspirate $\left(>1.0 \times 10^{6} \mathrm{CFU} / \mathrm{mL}\right)$. Of the 4 patients only colonized with CRAb (not infected), 2 survived. A colonized patient can serve as a source or reservoir and thus can increase the spread of CRAb. CRAb colonization may prolong the hospital stay and increase medical costs and the ICU mortality rate. ${ }^{6}$ Of the 10 patients with VAP or bacteremia, 7 died. Our findings support a previous report associating CRAb infection in COVID-19 patients with worse outcomes. ${ }^{3}$

All isolates proved to be resistant to penicillins, cephalosporins, aminoglycosides, fluoroquinolones, and carbapenems, greatly limiting options for treatment. All patients with CRAb infection were previously treated with azithromycin, ceftriaxone, and piperacillin/tazobactam. Of these, 1 (patient 5) died before starting appropriate antibiotic treatment, 5 (patients 1, 3, 6, 11 and 14) received polymyxin monotherapy, and 4 received combination therapy. Of the 4 patients treated with combination therapy, 2 (patients 8 and 13) received polymyxin B plus meropenem; 1 (patient 4 ) received polymyxin $B$, meropenem, and vancomycin; and 1 (patient 9 ) received meropenem and vancomycin. Only 1 (20\%) of 5 patients treated with polymyxin monotherapy survived; 2 (50\%) of 4 patients who received combination therapy recovered.
The best treatment for CRAb infections is a matter of debate. Although polymyxin monotherapy is widely used against CRAb infections, combination therapy has been associated with higher probabilities of therapeutic success. ${ }^{7}$ Our results suggest that combination therapy may be more effective in treating COVID-19 patients with CRAb infection, although further studies are needed to evaluate this possibility.

ERIC-PCR results showed a monoclonal spread of CRAb in the COVID-19 ICU within a short period, characterizing an outbreak. The band profile of these isolates showed $100 \%$ similarity to representatives of an endemic CRAb clone (previously reported). ${ }^{8,9}$ This CRAb clone has been a persistent problem in our region since 2004, and although the newly opened ICU may have initially been contamination free, the clone spread rapidly in this unit. A. baumannii can survive for long periods on surfaces, including dry surfaces and human skin, which could facilitate its persistence and spread in ICUs. ${ }^{7}$ CRAb cross transmission between equipment (eg, ventilators, infusion pumps, and hemodialysis machines) and COVID-19 patients may also partly explain the onset of this outbreak. Furthermore, in several countries, including Brazil, health personnel were hired on an emergency basis to respond to the COVID-19 pandemic, impeding adequate training in infection prevention and control.

In our hospital, stricter barrier measures were implemented, increasing the effectiveness of screening and surveillance for $\mathrm{CRAb}$. The active surveillance culture and efficient performance of a multidisciplinary team were highly important in detecting and controlling the CRAb outbreak in the COVID-19 ICU.

In conclusion, constant infection-control measures are necessary to stop the spread of CRAb in the hospital environment, 
prevent outbreaks, and lower mortality rates, especially in this time of the SARS-CoV-2 pandemic. With overloaded health systems and shortages of health workers trained in infection management, as well as medical consumables and equipment, the best preventive measure remains changing gloves and hand washing.

Acknowledgments. The authors thank Dr Janet W. Reid for her English text review.

Financial support. This study was financed in part by the Coordenação de Aperfeiçoamento de Pessoal de Nível Superior, Brazil (CAPES), Finance Code 001. These government funds covered only the cost of laboratory materials and had no role in the study design or the decision to submit the work for publication.

Conflicts of interest. All authors report no conflicts of interest relevant to this article.

\section{References}

1. Grasselli G, Zangrillo A, Zanella A, et al. Baseline characteristics and outcomes of 1591 patients infected with SARS-CoV-2 admitted to ICUs of the Lombardy Region, Italy. JAMA 2020;323:1574-1581.
2. Lima WG, Brito JCM, Nizer WSC. Ventilator-associated pneumonia (VAP) caused by carbapenem-resistant Acinetobacter baumannii in patients with COVID-19: two problems, one solution? Med Hypotheses 2020;144:110139.

3. Sharifipour E, Shams S, Esmkhani M, et al. Evaluation of bacterial coinfections of the respiratory tract in COVID-19 patients admitted to ICU. BMC Infect Dis 2020;20:464.

4. Gottesman T, Fedorowsky R, Yerushalmi R, et al. An outbreak of carbapenem-resistant Acinetobacter baumannii in COVID-19 dedicated hospital. Infect Prev Pract 2021;3:100113.

5. Silbert S, Pfaller MA, Hollis RJ, et al. Evaluation of three molecular typing techniques for nonfermentative gram-negative bacilli. Infect Control Hosp Epidemiol 2004;25:847-851.

6. Lee $\mathrm{H}$, Lee $\mathrm{H}$. Clinical and economic evaluation of multidrug-resistant Acinetobacter baumannii colonization in the intensive care unit. Infect Chemother 2016;48:174-180.

7. Weinberg SE, Villedieu A, Bagdasarian N, et al. Control and management of multidrug resistant Acinetobacter baumannii: a review of the evidence and proposal of novel approaches. Infect Prev Pract 2020;2:100077.

8. Saalfeld SMS, Viana GF, Siqueira VLD, et al. Endemic carbapenem-resistant Acinetobacter baumannii in a Brazilian intensive care unit. J Hosp Infect 2009;72:365-368.

9. Moreira RRB, Viana GF, Moraes ACC, et al. Dissemination of Acinetobacter baumannii OXA-23 in old and new intensive care units without transfer of colonized patients. Infect Control Hosp Epidemiol 2018;39:1135-1137.

\title{
Extensive environmental contamination and prolonged severe acute respiratory coronavirus-2 (SARS CoV-2) viability in immunosuppressed recent heart transplant recipients with clinical and virologic benefit with remdesivir
}

\author{
Irina Rajakumar BScPhm, ACPR ${ }^{1}$ (1), Debra L. Isaac MD ${ }^{1,2}$, Nowell M. Fine MD ${ }^{1,2}$, Brian Clarke MD ${ }^{1,2}$, \\ Linda P. Ward $\mathrm{MLT}^{1}$, Rebecca J. Malott PhD², Kanti Pabbaraju MSc ${ }^{4}$, Kara Gill BSc${ }^{4}$, Byron M. Berenger MSc, MD ${ }^{2,4}$, \\ Yi-Chan Lin PhD ${ }^{3}$, David H. Evans PhD ${ }^{3, a}$ and John M. Conly MD ${ }^{1,2, a}$ (i) \\ ${ }^{1}$ Alberta Health Services, Calgary, Alberta, Canada, ${ }^{2}$ University of Calgary, Calgary, Alberta, Canada, ${ }^{3}$ University of Alberta, Edmonton, Alberta, Canada and \\ ${ }^{4}$ Alberta Public Health Laboratory, Alberta Precision Laboratories, Calgary, Alberta, Canada
}

To the Editor-Remdesivir is an antiviral medication that exhibits antiviral activity versus SARS-CoV-2, ${ }^{1-3}$ but in clinical trials, it has demonstrated conflicting results with respect to mortality in patients with severe coronavirus disease 2019 (COVID-19).,5 The use of remdesivir in immunosuppressed patients, including the initial posttransplant period with its high degree of immunosuppression, has not been well studied. ${ }^{6-8}$ We examined the virologic and clinical responses to remdesivir in 2 recent cardiac transplant cases with SARS-CoV-2 infection.

\footnotetext{
Author for correspondence: Dr John Conly, E-mail: john.conly@albertahealthservices. ca or jconly@ucalgary.ca. Or Dr David Evans, E-mail: devans@ualberta.ca

aSenior authors of equal contribution.

Cite this article: Rajakumar I, et al. (2022). Extensive environmental contamination and prolonged severe acute respiratory coronavirus-2 (SARS CoV-2) viability in immunosuppressed recent heart transplant recipients with clinical and virologic benefit with remdesivir. Infection Control \& Hospital Epidemiology, 43: 817-819, https:// doi.org/10.1017/ice.2021.89
}

\section{Methods}

Nasopharyngeal (NP) swabs, saliva, and clinical and environmental samples were collected at regular intervals beginning shortly after admission. They were tested using molecular assays ${ }^{9}$ and quantitative culture (Supplementary Material online). Patients provided informed consent with the approval of the University of Calgary's Health Research Ethics Board (no. 20-0444).

\section{Case descriptions}

Case 1 was a 56-year-old woman with a history of dilated cardiomyopathy with end-stage heart failure, type- 2 diabetes mellitus, hypothyroidism, osteoporosis, and anemia. This patient underwent an orthotopic heart transplant with antithymocyte globulin (ATG) induction and standard triple immunosuppressive therapy. The patient was discharged 30 days later with prednisone, tacrolimus, and mycophenolate mofetil (MMF), and standard prophylactic medications.

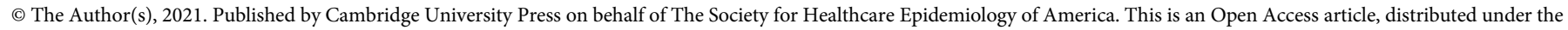
terms of the Creative Commons Attribution licence (http://creativecommons.org/licenses/by/4.0/), which permits unrestricted re-use, distribution, and reproduction in any medium, provided the original work is properly cited. 